International Journal of Pure and Applied Mathematics

Volume 87 No. 4 2013, 513-528

ISSN: 1311-8080 (printed version); ISSN: 1314-3395 (on-line version)

url: http://www.ijpam.eu

doi: http://dx.doi.org/10.12732/ijpam.v87i4.2

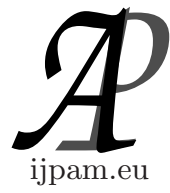

\title{
STRONG RECONSTRUCTIBILITY OF THE BLOCK-CUTPOINT TREE
}

\author{
Martin Dowd \\ 1613 Wintergreen Pl. \\ Costa Mesa, CA 92626, USA
}

\begin{abstract}
It is shown that the multiset of reconstruction trees of the connected components of a graph is strongly reconstructible. It is then shown, that an annotated version of the block-cutpoint tree is strongly reconstructible. A refinement of this result is given. Some cases of reconstructibility of separable graphs are given. A conjecture which implies the graph reconstruction conjecture is given.
\end{abstract}

AMS Subject Classification: $05 \mathrm{C} 60$

Key Words: strong reconstructibility, reconstruction tree, separability

\section{Introduction}

The graph reconstruction conjecture states that a graph (with at least 3 vertices) is determined by the multiset of its point deleted subgraphs (called the "deck"). This problem was first formulated in 1941, and has remained open since. Many classes of graphs and properties of graphs are known to be reconstructible [3]. It is still open whether separable graphs are reconstructible, but some special cases are known [1].

R. Statman [5] considers graphs $G$ all of whose induced subgraphs are reconstructible. While disconnected graphs are reconstructible, for example, whether they are in this category is equivalent to the graph reconstruction conjecture.

Received: March 29, 2013

(c) 2013 Academic Publications, Ltd. url: www.acadpubl.eu 
Statman also defines the reconstruction tree of a graph, and shows that $G$ is determined by its tree if its subgraphs are reconstructible.

We define $G$ to be strongly reconstructible if it is determined by its reconstruction tree. All graphs are strongly reconstructible iff they are all reconstructible, but on a graph by graph basis strong reconstructibility is a stronger statement.

While it is not immediate that disconnected graphs are strongly reconstructible, we show in section 3 that the reconstruction trees of the components are determined by the reconstruction tree of the graph, thereby reducing the reconstruction conjecture to strong reconstructibility of connected graphs.

In section 4 it is shown that the block-cutpoint tree of a connected graph is strongly reconstructible. In fact, it is shown that the " $\beta$-tree", which is the block-cutpoint tree with reconstruction trees given for the blocks. is strongly reconstructible.

In section $5, \beta^{+}$-trees are defined, as $\beta$-trees with some additional annotation. It is shown that the $\beta^{+}$-tree is strongly reconstructible. In section 6 , some cases of reconstruction of separable graphs are proved, which are not in [1]. In section $7, \beta^{*}$ trees are defined, and the strong reconstructibility of these is shown to be equivalent to the graph reconstruction conjecture.

In an appendix, a proof of the strong reconstructibility of trees is given, for use in section 4 .

\section{Reconstruction Trees}

Given $n \geq 2$, let $\breve{T}$ be the rooted, directed tree of height $n-2$, where the vertices at depth $i<n-2$ have $n-i$ sons. Let $E$ denote the edge set of $\breve{T}$. Let $N$ denote a set of $n$ integers, to be used as edge labels; often $N=\{1, \ldots, n\}$. By an edge labeling of $\breve{T}$ is meant a map $\eta \mapsto N$, such that for each vertex $v$ of $\breve{T}$, the labels of the edges descending from $v$ are those not occurring on the path from the root to $v$. Let $L$ denote the set of leaves of $\breve{T}$. By a leaf labeling of $\breve{T}$ is meant a function $\lambda: L \mapsto\{0,1\}$. An edge labeling and a leaf labeling are said to be consistent if for any branch $b$, the leaf label at the end of $b$ depends only on the set of edge labels along $b$.

Say that a leaf label is legitimate if there is some edge labeling which it is consistent with. If $n=3$ all leaf labelings are legitimate, but this is not the case in general. Firstly the number of 1's must be divisible by $(n-2)$ !, but this is not sufficient. Indeed, if $n=4$, consider the sorted list of the number of leaves labeled 1 in each subtree of the root. There are 35 possible lists; 19 of 
these have even sum, but only 11 are legitimate.

By a labeled graph $G$ is meant an $n$ vertex graph whose vertices are labeled with the distinct elements of the edge label set $N$. Given an edge labeling $\eta$, each branch $b$ of $\breve{T}$ determines a pair $\{u, v\}$ of vertex labels, namely those omitted along the branch. This yields a bijective correspondence between the labeled graphs $G$ and the leaf labelings $\lambda$ consistent with $\eta$. The leaf label at the end of a branch $b$ equals 1 if $\{u, v\}$ is an edge of $G$, and 0 if it is not. $G(\eta, \lambda)$ will be used to denote the labeled graph determined by $\eta$ and $\lambda$.

Let $\Gamma$ be the automorphism group of $\breve{T}$. Letting $V$ denote the set of vertices of $\breve{T}$, an element of $\Gamma$ is a bijection $\gamma: V \mapsto V$ with certain properties, so that bijections $L \mapsto L$ and $E \mapsto E$ are induced, which may also be denoted $\gamma$. For an edge labeling $\eta$, an element $\gamma \in \Gamma$ induces a new edge labeling $\eta^{\gamma}$ by "moving" the label, so that $\eta^{\gamma}(\gamma(e))=\eta(e)$ for $e \in E$. Similarly, given a leaf labeling $\lambda$, $\gamma$ induces a new labeling $\lambda^{\gamma}$.

Given an edge labeling $\eta_{1}$, there is a bijective correspondence from an edge labeling $\eta_{2}$ to the tree automorphism $\gamma_{\eta_{1} \mapsto \eta_{2}}$ which moves $\eta_{1}$ to $\eta_{2}$.

Theorem 1. Suppose $\eta$ is an edge labeling, $\lambda$ is a leaf labeling, and $\gamma \in \Gamma$.

a. If $\eta$ is consistent with $\lambda$ then $\eta^{\gamma}$ is consistent with $\lambda^{\gamma}$, and $G\left(\eta^{\gamma}, \lambda^{\gamma}\right)=$ $G(\eta, \lambda)$.

b. If $G\left(\eta_{1}, \lambda_{1}\right)=G\left(\eta_{2}, \lambda_{2}\right)$ then $\lambda_{2}=\lambda_{1}^{\gamma_{\eta_{1} \mapsto \eta_{2}}}$.

c. If $\pi$ is an isomorphism from $G\left(\eta_{1}, \lambda_{1}\right)$ to $G\left(\eta_{2}, \lambda_{2}\right)$ then $G\left(\pi \circ \eta_{1}, \lambda_{1}\right)=$ $G\left(\eta_{2}, \lambda_{2}\right)$.

Proof. For part a, if $b$ is a branch with endpoint $v$ then the vertex at the end of $\gamma(b)$ is $\gamma(v)$. Part $\mathrm{b}$ follows by part a and the fact that $\eta_{1}^{\gamma \eta_{1} \mapsto \eta_{2}}=\eta_{2}$. Part c follows, because the change of names to $\eta_{1}$ is exactly what is required to produce the same labeled graph.

A pair $\langle\breve{T}, \lambda\rangle$ where $\lambda$ is a legitimate leaf labeling will be called a reconstruction tree, and frequently denoted $T$. The abbreviation " $\rho$-tree" for reconstruction tree will be used. An isomorphism from a $\rho$-tree $\left\langle\breve{T}, \lambda_{1}\right\rangle$ to a $\rho$-tree $\left\langle\breve{T}, \lambda_{2}\right\rangle$ is a $\gamma \in \Gamma$ such that $\lambda_{2}=\lambda_{1}^{\gamma}$.

A graph $G$ is said to be consistent with a $\rho$-tree $T$ if $T$ has an edge labeling $\eta$ which is consistent with $\lambda$, such that $G$ is the underlying graph of $G(\eta, \lambda)$. By theorem 1 each nonempty graph is consistent with a unique $\rho$-tree, up to isomorphism, provided the one point graph is considered to have a special $\rho$ tree (the empty tree). As shown in [5], the graph reconstruction conjecture is equivalent to the statement that distinct graphs have distinct $\rho$-trees. Say 
that a function or predicate on graphs is strongly reconstructible if its value is determined by the $\rho$-tree of the graph.

It follows by theorem 1 that each graph which is consistent with an isomorphic copy of a $\rho$-tree $T$ is consistent with $T$; thus, $\lambda$ can be fixed. For a leaf labeling $\lambda$, let $\Gamma_{\lambda}$ denote $\left\{\gamma \in \Gamma: \lambda^{\gamma}=\lambda\right\}$.

\section{Theorem 2.}

a. $G\left(\eta^{\gamma}, \lambda\right)=G(\eta, \lambda)$ iff $\gamma \in \Gamma_{\lambda}$.

b. A permutation $\pi$ of $N$ is an isomorphism from $G\left(\eta_{1}, \lambda\right)$ to $G\left(\eta_{2}, \lambda\right)$ iff $\eta_{2}=$ $\eta_{1}^{\gamma \circ \gamma_{\eta_{1} \mapsto \pi \circ \eta_{1}}}$ for some $\gamma \in \Gamma_{\lambda}$.

Proof. If $\gamma \in \Gamma$ then $G\left(\eta^{\gamma}, \lambda\right)=G(\eta, \lambda)$ follows by theorem 1.a. If $G\left(\eta^{\gamma}, \lambda\right)=$ $G(\eta, \lambda)$ then $G\left(\eta^{\gamma}, \lambda^{\gamma}\right)=G\left(\eta^{\gamma}, \lambda\right)$, so $\lambda^{\gamma}=\lambda$. This proves part a. For part $\mathrm{b}, \pi$ is an isomorphism iff $G\left(\eta_{2}, \lambda\right)=G\left(\pi \circ \eta_{1}, \lambda\right)$. By part a this is so iff $\gamma_{\pi \circ \eta_{1} \mapsto \eta_{2}} \in \Gamma_{\lambda}$. The result follows, since $\gamma_{\eta_{1} \mapsto \eta_{2}}=\gamma_{\pi \circ \eta_{1} \mapsto \eta_{2}} \circ \gamma_{\eta_{1} \mapsto \pi \circ \eta_{1}}$.

\section{Disconnected Graphs}

In this section it is shown that the $\rho$-trees of the components of a graph $G$ are determined by the $\rho$-tree of $G$, and conversely.

Lemma 3. The number of vertices $v(G)$ and the number of edges $e(G)$ are strongly reconstructible.

Proof. If the tree is of height $h, v(G)=h+2$. The number of edges is obtained by dividing the number of leaves labeled 1 by $h$ !.

Define the size of a graph to be the number of vertices. A vertex $v$ may be "indicated" in a $\rho$-tree $T$, by labeling an edge out of the root with $v$. In this case let $T_{v}$ denote the subtree rooted at the end of the edge.

Lemma 4. If a vertex $v$ is indicated, its degree is determined. A fortiori the degree sequence is strongly reconstructible.

Proof. The degree of $v$ equals $e(T)-e\left(T_{v}\right)$, where $e(T)$ is the number of edges in any graph consistent with $T$.

Lemma 5. The multiset of component sizes is strongly reconstructible. 
Proof. We may assume the multiset is a sequence, with sizes in nonincreasing order. The proof is by induction on the number of vertices $n$. The basis case $n=1$ is trivial. If there is an isolated vertex $v$, extend the sequence for $T_{v}$ with an additional element of value 1 . Otherwise say that a vertex $v$ is nonseparating if its removal does not increase the number of components. Each component contains a nonseparating vertex (in fact at least 2; consider a spanning tree). The number of components for $T$ is determined inductively, since it is the minimum such among the $T_{v}$. Further, whether $v$ is nonseparating is determined. For each nonseparating vertex $v$, consider the sequence of component sizes for $T_{v}$. Consider the sequence which is highest in the lexicographic order. The list for $T$ is obtained by adding 1 to its last element.

If $S$ and $T$ are multisets let $S+T$ denote the multiset containing $x s+t$ times, where $S$ contains $x s$ times and $T$ contains $x t$ times.

Lemma 6. Given multisets $S_{1}, \ldots, S_{k}$ and $S_{1}+U, \ldots, S_{k}+U, U$ is determined.

Proof. Let $T=S_{1}+\cdots+S_{k}$. Suppose $S_{i}+U=S_{\pi(i)}+V, 1 \leq i \leq k$, where $\pi$ is a permutation. Then $T+k U=T+k V$, so $U=V$.

Theorem 7. The $\rho$-trees of the components are strongly reconstructible.

Proof. The proof is by induction on the number of vertices $n$. The basis case $n=1$ is trivial. If there is only 1 component, the theorem is trivial. If there is an isolated vertex $v$, add a $\rho$-tree for a single vertex graph to the list of $\rho$-trees for the components for $T_{v}$. Otherwise, let $m$ be the minimum size of a component. It is easily seen using lemma 5 that it is determined if a vertex $v$ is in a component of size $m$. Choose such a $v$; the $\rho$-trees for $T$ of components of size greater than $m$ are those for $T_{v}$. Let $r$ be the number of components of size $m$. If $r>1$, consider the $\rho$-trees of the $m$ vertex components for the $T_{u}$, where $u$ ranges over vertices in a component of size $m$. Each $\rho$-tree of an $m$ vertex component appears $(r-1) n$ times. If $r=1$ choose a vertex $v$ in the component of size $m$. It is known if $u$ is in a component of size greater than $m$, for either $T$ or $T_{v}$. For each such $u$, the $m$-vertex $\rho$-trees other than the missing one for $T_{v}$ are known, by the induction hypothesis applied to $T_{v u}$. Also for each such $u$ all the $m$-vertex $\rho$-trees are known, by the induction hypothesis applied to $T_{u}$. The theorem follows by lemma 6 .

Let $N_{1}, \ldots, N_{l}$ be a sequence of nonempty subsets of the edge label set $N$, such that $N$ is the disjoint union of the $N_{i}$. Let $\breve{T}_{i}$ be the unlabeled tree for 
$\left|N_{i}\right|$ vertices. Choose a labeling $\eta$ for $\breve{T}$ with label set $N$, and for each $i$ choose a labeling $\eta_{i}$ for $\breve{T}_{i}$ with label set $N_{i}$.

To each leaf of $\breve{T}_{i}$ add a tree with two branches of length 2 , and extend $\eta_{i}$ so that the new edges below a leaf of $\breve{T}_{i}$ are labeled in each possible way with the two label values omitted along the branch to the leaf. As a special case, an empty tree becomes a single edge. Let $T_{i}^{E}$ extended the extended tree and $\eta_{i}^{E}$ the extended labeling.

A map $\chi_{\eta,\left\langle\eta_{i}\right\rangle}$, or simply $\chi$, may be defined from paths $p$ in $\breve{T}$, to sequences of paths $p_{1}, \ldots, p_{l}$ where $p_{i}$ is a path in $\breve{T}_{i}^{E}$. A path may be identified with the sequence of edge labels along the path. $\chi$ is defined recursively. If $p$ is the path of length 0 then so is each $p_{i}$. If $p$ is $q$ followed by $v$, then $p_{i}$ is $q_{i}$, followed by $v$ if $v \in N_{i}$.

Theorem 8. The $\rho$-tree of a graph is determined by the $\rho$-trees of its components.

Proof. Let $T_{1}, \ldots, T_{r}$ be the trees of the components. As in the remarks preceding the theorem, choose $N_{i}$ of size $n_{i}$ where $n_{i}$ is the number of vertices for $T_{i}$; choose $\eta_{i}$ and $\eta$; and let $\chi$ denote $\chi_{\eta,\left\langle\eta_{i}\right\rangle}$. Letting $\lambda_{i}$ denote the leaf labeling of $T_{i}$, a leaf labeling $\lambda$ of $\breve{T}$ is determined by $\chi$; the label at the end of a branch is 0 if the omitted labels are in different $T_{i}$, else the label at the end of the branch in that $T_{i}$ where the two labels are omitted. Note that $G(\eta, \lambda)$ is the union of the $G\left(\eta_{i}, \lambda_{i}\right)$. Suppose now that $\eta_{i}^{\prime}$ are some other consistent labelings of the $T_{i}$. There is a unique $\eta^{\prime}$ such that $\chi_{\eta^{\prime},\left\langle\eta_{i}^{\prime}\right\rangle}=\chi$, namely, if the edge $e$ corresponds to $f$ in $T_{i}$ under $\chi$, let $\eta^{\prime}(e)=\eta_{i}^{\prime}(f)$. It follows that $G(\eta, \lambda)$ is the union of the $G\left(\eta_{i}^{\prime}, \lambda_{i}\right)$. This proves the theorem.

By theorem 7 there is a function $[T] \mapsto\left\{\left[T_{1}\right], \ldots,\left[T_{l}\right]\right\}$ from the equivalence class of a $\rho$-tree to the multiset of equivalence classes of the $\rho$-trees of the connected components of any graph consistent with $T$. To compute the value at $T$, let $G$ be any graph consistent with $T$, let $G_{1}, \ldots, G_{l}$ be its components, and let $T_{i}$ be the $\rho$-tree for $G_{i}$. By theorem 8 there is a function $\left\{\left[T_{1}\right], \ldots,\left[T_{l}\right]\right\} \mapsto$ $[T]$. To compute the value at $T_{1}, \ldots, T_{l}$, let $G_{1}, \ldots, G_{l}$ be any graphs where $G_{i}$ is consistent with $T_{i}$, let $G$ be the graph with components $G_{1}, \ldots, G_{l}$, and let $T$ be the $\rho$-tree for $G$. It is readily seen that these functions are inverse to each other.

Theorem 9. Suppose $T$ is a $\rho$-tree and $T_{1}, \ldots, T_{l}$ are the $\rho$-trees of the components. If a vertex $v$ is indicated in $T$ it may be indicated among the $T_{i}$.

Proof. Let $A$ be the component trees of $T$ and let $B$ be the component trees 
of $T_{v}$. Let $i$ be such that $T_{i}$ is the single $\rho$-tree of $A$ which is missing from $B$. Let $C=B-(T-A)$. By theorem $8 T_{i v}$ is determined by $C$.

\section{4. $\beta$-Trees}

In this section, unless otherwise noted, a graph is connected. The block-cutpoint tree of a graph is a tree, with some vertices corresponding to cutpoints, and some to blocks (see [4]). We define the $\beta$-tree of a graph to be the block-cutpoint tree, with the vertex for a block labeled with the $\rho$-tree of the block. We will say that the $\beta$-tree is inductively determined for a $\rho$-tree $T$ if it is determined by $T$ and a set of $\beta$-trees for the $T_{v}$, derived from some graph consistent with $T$. Clearly, if the $\beta$-tree is inductively determined for all $T$ then it is determined for all $T$.

Say that a vertex $v$ is indicated in a tree if the tree is equipped with a $0-1$ labeling of the vertices which is 1 only at $v$. Define a twig of a graph $G$ to be an edge incident to a vertex of degree 1 . If $G$ is not a tree, the trunk of $G$ is defined to be the graph obtained by successively removing twigs until none remain. Define a limb of $G$ to be a maximal subtree, with the vertex contained in the trunk indicated. Note that $G$ is the trunk, with the limbs attached at distinct vertices. A block is said to be terminal if it contains only 1 cutpoint.

The determination of the $\beta$-tree will be divided into 4 cases, where a case holds only if lower numbered cases do not hold.

1. There are no vertices of degree 1.

2. The trunk consists of a single block.

3. There are terminal blocks which are not edges.

4. All terminal blocks are edges.

Lemma 10. In case 1, the $\beta$-tree is inductively determined.

Proof. The least size $n$ of a terminal block can be determined by considering the least size of a component in a graph for $T_{v}$ for cutpoints $v$. Suppose $v$ is a non-cutpoint vertex. For a cutpoint $u$ of $T_{v}$ let $A$ be the subtree of the $\beta$-tree for $T_{v}$, determined by paths from $u$ to a terminal block of size less than $n$. Say that $u$ is a candidate if $A$ has no neighbors outside $A$, other than at vertex $u$; and $A$ (with $u$ deleted if it is "dangling") is the $\beta$-tree of a graph of size $n$. It is easy to see that $T_{v}$ can have at most one candidate vertex. Also, if it does, then $v$ cannot be in a non-terminal block of size at most $n$. For $v$ such that there is a candidate vertex $u$, consider the sizes of blocks outside $A$, ordered in nonincreasing order. Call $u$ good if this sequence is highest in the lexicographic 
order among candidate $u$. It is readily seen that $u$ is good iff $v$ is in a terminal block of size $n$. If $u$ is good, the $\beta$-tree of $T$ is that of $T_{v}$, with $A$ replaced by a single block $B$ of size $n$ adjacent to $u$. To determine the $\rho$-tree of $B$, let $r$ be the number of terminal blocks of size $n$. If $r>1$, consider the $\rho$-trees of the $n$ vertex terminal blocks for the $T_{u}$, where $u$ ranges over non-cutpoint vertices in a terminal block of size $n$. Each $\rho$-tree of a $n$ vertex terminal block appears $(r-1)(n-1)$ times. If $r=1$ choose a vertex $v$ in the terminal block of size $n$. Let $m$ be the largest block size. It is known if $u$ is in a block of size $m$, for either $T$ or $T_{v}$. For each such $u$, the $n$ vertex $\rho$-trees other than the missing one for $T_{v}$ are known. Also for each such $u$ all the $n$ vertex trees are known. $B$ is determined by lemma 6 .

Lemma 11. The multiset of the limbs is inductively determined.

Proof. Let $T$ be the $\rho$-tree, and $G$ a consistent graph. If $G$ contains no vertices of degree 1 the claim is trivial. Let $v$ range over vertices degree 1 . If each $T_{v}$ has $n$ limbs, all of which are edges, then $G$ has $n+1$ limbs, all of which are edges. If some $T_{v}$ have $n$ limbs and some $n+1$, let $v$ be such that there are $n$; then the limbs of $T$ are those of $T_{v}$, with an additional edge. In the remaining case, for each $v$ consider the sequence of sizes of the limbs of $T_{v}$ in nonincreasing order. Let $v$ be such that this sequence is lexicographically highest, and let $n^{-}$be the least size in the sequence. Then the least size of a limb of $T$ equals $n=n^{-}+1$, the size sequence for $T$ equals that for $T_{v}$ with its last element replaced by $n$, and the limbs of $T$ of size greater than $n$ are those of $T_{v}$. Let $r$ be the number of limbs of size $n$. It is inductively determined whether $v$ is in a limb of size $n$. If $r>1$, the set $S$ of limbs of size $n$ is the union of the sets for $T_{v}$ for $v$ in a limb of size $n$. Let $s=|S|$. If $s=1$ the multiplicity of the single element of $S$ is $r$. If $s>1$ the multiplicity of an element of $S$ is the maximum over $v$ of its multiplicity in $T_{v}$.

If $r=1$ and there is more than one limb, choose a leaf $v$ in the limb of size $n$. Let $m$ be the largest size of a limb. It is known if $u$ is in a limb of size $m$, for either $T$ or $T_{v}$. For each such $u$, the $n$ vertex limbs other than the missing one for $T_{v}$ are known. Also for each such $u$ all the $n$ vertex limbs are known. The theorem follows in this case by lemma 6 .

In the remaining case of only one limb $L$, if $L$ has only one leaf, then $L$ is easily determined. Otherwise let $u$ be the vertex of $L$ in the trunk. Let $w$ be the closest vertex to $u$ which has degree greater than 2 . Let $e$ range over the edges incident to $w$, other than the edge on the path to $u$. Call the subtree consisting of all paths from $w$ to a leaf of $L$ containing a given $e$ a sublimb. The multiset of sublimbs may be determined by methods similar to methods already 
described. In particular, consider the cases where all sublimbs are edges, some are and some aren't, and all aren't. In the third case determine the sublimb size list. Let $n_{s}$ be the smallest size and $r_{s}$ the number of sublimbs of size $n_{s}$. consider the cases $r_{s}>1$ and $r_{s}=1$; when $r_{s}=1$ there is more than one sublimb.

Lemma 12. In case 2 , the $\beta$-tree is inductively determined.

Proof. Each limb may be converted to its $\beta$-tree, with an extra cutpoint vertex added if needed, and the cutpoint vertex for the root of the limb joined by an edge to the block vertex for the trunk.

Call a limb minimal if it consists of a single edge. By lemma 11 it is inductively determined whether a degree 1 vertex is the leaf of a minimal limb.

Lemma 13. It is inductively determined whether all terminal blocks are edges.

Proof. If the graph is a tree, or if there are no vertices of degree 1 , the result is trivial. Write $P(T)$ to denote that all terminal blocks are edges. Note that $P(T)$ iff every non-terminal block of the trunk has a limb attached to a vertex which is not a cutpoint of the trunk. If not all limbs are minimal, let $v$ be a leaf which is in a non-minimal limb; then $P(T)$ iff $P\left(T_{v}\right)$. Otherwise let $s$ be the number of limbs, and $t$ the number of terminal blocks in the trunk. If $s>t$ then $P(T)$ iff $P\left(T_{v}\right)$ for some degree 1 vertex $v$. If $s<t$ then $\neg P(T)$. If $s=t$ then $P(t)$ iff for every degree 1 vertex $v$, in the trunk of $T_{v}$, no non-terminal block has a limb attached, no cutpoint has a limb attached, and no terminal block has more than 1 limb attached.

If $u$ is a vertex in a tree, and $e$ is an edge incident to $v$, define the frond through $e$ to be the union of the set of branches from $u$ to a leaf, which contain $e$.

Lemma 14. In case 3 , the $\beta$-tree is inductively determined.

Proof. By lemmas 10 and 11, the $\rho$-trees of the blocks are inductively determined. Let $Q$ be the multiset of $\rho$-trees of the blocks of $T$. Let $W$ be the set of vertices which are non-cutpoints and have degree greater than $1 ; v \in W$ iff $v$ is in the trunk, is not a cutpoint of the trunk, and has no limb attached. If $v \in W$ let $Q_{v}$ be the multiset of $\rho$-trees of the blocks of $T_{v}$. Let $B_{v}$ be the $\rho$-tree of $Q$ missing from $Q_{v} ; B_{v}$ is the block containing $v . X_{v}=Q_{v}-Q$ is the blocks into which $B_{v}$ is split by removing $v$. For $v \in W$ say that a cutpoint $u$ 
of $T_{v}$ is good if there is a set of fronds with union $A$, whose multiset of block $\rho$-trees equals $X_{v}$; and such that the multiset of block $\rho$-trees of $T_{v}$ outside $A$ equals $Q-B_{v}$. If there is a good vertex then it is unique. $T_{v}$ has a good vertex iff $v$ is in a terminal block. If $u$ is good, the $\beta$-tree of $T$ is that of $T_{v}$, with $A$ replaced by $B$.

Recall that a tree $H$ is the block-cutpoint tree of some connected graph iff every path between leaves has even length (see [4]); call such a tree a blockcutpoint tree. Suppose $H$ is a block-cutpoint tree and $v$ is a leaf. $H_{v}$ is a block-cutpoint tree, unless $v$ is adjacent to a vertex $w$ of degree 1 , in which case $H_{v w}$ is a block-cutpoint tree. Call $H_{v}$ or $H_{v w}$ the adjusted leaf-deleted subtree.

Lemma 15. A block-cutpoint tree $G$ with at least three vertices is determined by the multiset $D$ of its adjusted leaf-deleted subtrees.

Proof. The proof is obtained from the proof of theorem 24 given in the appendix, by making the necessary adjustments. First, $d$ is the maximum of the diameters of the subtrees in $D$, unless $D$ consists of two paths of length $d-2$. Also. the tree is always central. By a p-subtree is meant the adjusted leaf-deleted subtree. There are no changes in case 1 . In case 2 , there is a single p-subtree of diameter $d-2$; the argument is otherwise unchanged. If case 3 there are two p-subtrees of diameter $d-2$. A path of length 2 must be added to one end of $S$; which end is either determined or irrelevant.

Lemma 16. In case 4 , the $\beta$-tree is inductively determined.

Proof. By definition of case 4 , the block-cutpoint tree has at least 3 vertices. Inductively the adjusted leaf-deleted subtrees of the $\beta$-tree are determined. By lemma 15 the block-cutpoint is determined. In fact, it is easy to see that the $\beta$-tree is inductively determined. The fronds as in the proof of theorem 24 with the blocks labeled with their $\beta$-trees are determined. In case 3 , the block added to the $\beta$-tree is an edge.

Theorem 17. The $\beta$-tree is strongly reconstructible.

Proof. This follows by lemmas 10, 12, 14, and 16 . 


\section{5. $\beta^{+}$-Trees}

The $\beta^{+}$-tree of a graph is defined to be the $\beta$-tree, with the cutpoint vertices labeled with distinct labels, and the cutpoints adjacent to a block vertex indicated in the $\rho$-tree of the block, provided the block has at least 3 vertices.

Theorem 18. The $\beta^{+}$-tree is strongly reconstructible.

Proof. The modifications needed to the proof of theorem 17 will be given. For lemma 10 it is necessary to determine $B$, with its cutpoint indicated. The method is identical. For lemma 12, there are three subcases. In case 2a, the set of limbs consists of 1 minimal limb. Let $u$ be the degree 1 vertex of the minimal limb and $v$ the cutpoint. It suffices to show that $v$ can be indicated in $T_{u}$. In $T_{v} u$ can be found. Choose some son of the root of $T_{u}$ whose subtree is isomorphic to $T_{v u}$; it is irrelevant which is chosen. In case $2 \mathrm{~b}$, the set of limbs consists of $s>1$ minimal limbs. By deleting all but 1 degree 1 vertex in each possible order, each $\rho$-tree for the trunk, with 1 cutpoint indicated, occurs $(s-1)$ ! times. The $\rho$-tree of the trunk, with each cutpoint indicated, is easily determined. In case $2 \mathrm{c}$, there are non-minimal limbs. The $\rho$-tree of the trunk with the cutpoints indicated may be determined by considering the $\beta^{+}$-tree for $T_{v}$, where $v$ is any degree 1 vertex which is not the endpoint of a minimal limb. For lemma $14 B$ with its cutpoint indicated is inductively determined.

For lemma 16, in cases 1 and 2 of theorem 24 let $S$ be the set of fronds of the block-cutpoint tree at the center. The fronds with the cutpoints indicated in the block are inductively determined. If the central node is a block node it remains to indicate the cutpoints in its $\rho$-tree. The case where all elements of $S$ have 3 vertices is included in case $2 \mathrm{~b}$. For the case where some elements of $S$ have 3 vertices and some have more, deleting a leaf of a terminal edge yields the central block, with a 3 vertex frond missing. If there is a frond with more than 4 vertices, deleting a leaf of such gives the 3 vertex fronds. Otherwise, deleting a leaf of a 4 vertex frond results in a missing $T_{v}$ of the 4 vertex fronds, and this can be removed from the $T_{w}$ for the 3 vertex fronds. If all fronds in $S$ have more that 3 vertices, delete a leaf of one with the smallest number of vertices. For lemma 16 in case 3 of theorem 24, both the old end block and the new block are edges.

Define a pre- $\beta^{+}$-tree to be a block-cutpoint tree, annotated as follows:

- The cutpoint vertices are labeled with distinct integers from an $n$ element label set $N$; let $N_{c}$ denote the set of cutpoint labels. 
- Block vertex $i$ is labeled with $\breve{T}_{i}$ for $n_{i}$ vertices, where $n_{i} \geq d_{i}$ where $d_{i}$ is the degree of the vertex.

- The cutpoints adjacent block $i$ are indicated in $\breve{T}_{i}$.

- Block $i$ is assigned a label set $N_{i}^{-}$, where these form a partition of $N-N_{c}$; let $N_{i}$ denote $N_{i}^{-}$together with the labels of the cutpoints adjacent to block $i$.

An edge labeling $\eta_{\Theta}$ of a pre- $\beta^{+}$-tree $\Theta$ is a sequence $\eta_{i}$, where $\eta_{i}$ is an edge labeling of $\breve{T}_{i}$ with label set $N_{i}$, which respects the indications of the cutpoints. $\Theta$ may be extended as before, and its edge labelings also.

Let $\breve{T}$ be the tree for $n$ vertices, $N$ a label set of size $n, \eta$ an edge labeling of $\breve{T}, \Theta$ a pre- $\beta$-tree, and $\eta_{\Theta}$ an edge labeling of $\Theta$. A map $\chi_{\eta \mapsto \eta_{\Theta}}$, or simply $\chi$, will be defined, similarly to that for component trees. In the recursion step, if $p$ is $q$ followed by $v$, then $p_{i}$ is $q_{i}$, followed by $v$ if $v \in N_{i}$; note that if $v$ is a cutpoint then more than one $p_{i}$ will be extended.

As before, if $b$ is a branch of $\breve{T}$ omitting $\{u, v\}, u$ and $v$ are in the same $N_{i}$ iff in $\chi(b), p_{i}$ is of length $n_{i}-2$. However the $p_{j}$ for $j \neq i$ have length $n_{j}$ or $n_{j}-1$, the latter occurring iff $u$ or $v$ is a cutpoint of block $j$. Note that a pair of cutpoints can occur in at most one block.

Theorem 19. The $\rho$-tree is determined by the $\beta^{+}$-tree.

Proof. Given a $\beta^{+}$tree, with block leaf labelings $\lambda_{i}$, choose edge labelings for the block $\rho$-trees $T_{i}$ which are consistent with the $\lambda_{i}$. This determines a labeled graph $G$. Choose an edge labeling $\eta$ for $\breve{T}$. Let $\lambda$ be the leaf labeling of $\breve{T}$ corresponding to $G$. Let $\chi$ be as described in remarks preceding the lemma. Suppose now that $\eta_{i}^{\prime}$ are some other consistent edge labelings of the block $\rho$-trees $T_{i}$. There is a unique $\eta^{\prime}$ such that $\chi_{\eta^{\prime}, \eta_{\Theta}^{\prime}}=\chi$, namely, if the edge $e$ corresponds to $f_{1}, \ldots, f_{s}$ under $\chi$ where $f_{j}$ is in $T_{i_{j}}$, then all $\eta_{i}\left(f_{j}\right)$ are equal; let $\eta^{\prime}(e)$ be their common value. It follows that $G(\eta, \lambda)$ is the union of the $G\left(\eta_{i}^{\prime}, \lambda_{i}\right)$ with cutpoints identified. This proves the theorem.

\section{Some Cases of Reconstructibility}

If the deck $\left\{G_{v}\right\}$ of a graph $G$ is given, then the $\rho$-tree for $G$ is the $\rho$-tree for $v(G)$ vertices, where the subtrees of the root are the $\rho$-trees of the $G_{v}$. Also, the $\beta$-trees of the $G_{v}$ are known, whence the $\beta$-tree of $G$ is. Other results about $\rho$-trees can similarly be adapted; for example lemma 11 may be adapted to show that the limbs are known if the deck is known. This was already shown in [1], and also case 1 of the following. 
Theorem 20. A separable connected graph is reconstructible in cases 1, 3 , and 4 .

Proof. In case 1 , the $v$ such that $G_{v}$ has a good vertex $u$ may be determined, as in the proof of lemma 10; the good vertex may be located in $G_{v}$. The missing block $B$ with the cutpoint marked may be determined, just as in lemma10. The proof for case 3 is similarly obtained by adapting the proof of lemma 14 . In case 4 , the $\beta$-tree is known. Let $n$ be the least size of a terminal block in the trunk. It may be determined whether a vertex $v$ is a vertex of such a block, other than the cutpoint vertex $u$ of the trunk. Also, the missing $\rho$-tree of the block together with the limbs attached to the block is known. It may be seen that $u$ may be determined in $G_{v}$. Also, the missing terminal block of the trunk may be determined, with its cutpoint in the trunk marked, and its attached limbs.

\section{7. $\beta^{*}$-Trees}

Let $W$ be a subset of the edge label set for a $\rho$-tree $T$. Define a complete indication of $W$ in $T$ to be the edges labeled with elements of $W$ in some consistent edge labeling of $T$. Say that a complete indication is determined if in any consistent labeling of $T$ there is a set of vertices $W^{\prime}$ such that there is a $\gamma \in \Gamma_{\lambda}$ which moves the edges labeled $W$ to the edges labeled $W^{\prime}$.

Theorem 21. Suppose $G$ is a labeled graph consistent with $T$ and let $\eta$ be the edge labeling of $T$ corresponding to $G$. $G$ is strongly reconstructible iff the complete indication of all edge labels as indicated by $\eta$ is determined.

Proof. This follows by theorem 2 .

Theorem 22. The $\beta^{*}$-tree is inductively determined in cases 1,3 , and 4 .

Proof. The proof in cases 1 and 3 is essentially the same as the proof of these cases in theorem 18. The method of theorem 20 can be used in case 4 (this could have been used in theorem 18 also).

Theorem 23. If the $\beta^{*}$-tree is strongly reconstructible then the graph reconstruction conjecture is true. 
Proof. Let $G$ be an inseparable graph. Construct $G^{\prime}$ by attaching an edge to each vertex of $G$. The $\beta^{*}$-tree of $G^{\prime}$ is determined. The $\rho$-tree of $G$ with every vertex completely indicated is known, whence by theorem $21 G$ is known. Now suppose $G$ is any graph. In its $\beta^{*}$-tree, the blocks are known. Further, the cutpoint vertices may be labeled in each block. Thus, $G$ is known.

\section{References}

[1] J.A. Bondy, On Ulam's conjecture for separable graphs, Pacific J. Math., 31 (1969), 281-288, doi: 10.2140/pjm.1969.31.281.

[2] J.A. Bondy, On Kelly's congruence theorem for trees, Jour. Camb. Phil. Soc., 65 (1969), 387-397, doi: 10.1017/S0305004100044364.

[3] J.A. Bondy, R.L. Hemminger, Graph reconstruction - A survey, J. Graph Theory, 1 (1977), 227-268, doi: 10.1002/jgt.3190010306.

[4] F. Harary, Graph Theory, Addison-Wesley (1971).

[5] R. Statman, Reductions of the graph reconstruction conjecture, Discrete Math., 36 (1981), 103-107, doi: 10.1016/0012-365X/881/990177-1.

\section{Appendix}

This section includes a proof that forests are strongly reconstructible, which is referred to in previous sections. It is essentially the same as that given in [2]. A graph is a forest iff its connected components are all trees. A connected graph is a tree iff $v=e+1$ where $e$ is the number of edges and $v$ the number of vertices. The claim thus follows by induction from the claim that a tree is determined by its deck. The reader is assumed to be familiar with the notion of the center or bicenter of a tree (see [4]).

Recall the definition in section 4 of a frond at a vertex in a tree. In this section the term will be restricted to mean a frond at the center of a central tree, or a the frond of a bicentral tree at a bicenter and other than that containing the central edge.

Theorem 24. A tree $G$ with at least two vertices is determined by the multiset $D$ of point-deleted subgraphs of its leaves. 
Proof. Define a p-vertex to be an endpoint of a path of length $d$ where $d$ is the diameter. Define a p-subtree to be the point deleted subtree of a p-vertex. Define a frond to be radial if it contains a p-vertex.

We show that the p-subtrees can be determined, and the tree can be reconstructed from these. The proof is broken in to several cases. First, note that $d$ is the maximum of the diameters of the subtrees in $D$, unless $D$ consists of two paths of length $d-1$. Also. the tree is central if $d$ is even, and bicentral iff $d$ is odd.

Case 1a: all subtrees in $D$ have diameter $d$ and $G$ is central. Let $i$ be the least number of p-vertices in a subtree in $D$; then the p-subtrees are those with $i$ p-vertices. Let $j$ be the least number of p-vertices of $G$ in a radial frond of $G$. For a p-subtree it may be determined whether the deleted vertex is in a $j$ vertex radial frond; let $E$ be these subtrees. Choosing any subtree in $E$, the radial fronds with more than $j$ p-vertices may be determined, each with the center marked. Let $k$ be the number of radial fronds with $j$ p-vertices. If $k>1$ then each radial frond with $j$ p-vertices occurs $(k-1) j$ times among the p-subtrees of $E$. If $k=1$ let $v$ be a vertex in the $j$ p-vertex radial frond. Let $u$ range over the p-vertices with more than $j$ p-vertices, in both $G$ and $G_{v}$. Determining the $j$ p-vertex radial fronds in each case, and applying lemma 6 , yields the $j$ p-vertex radial frond of $G$. If there is a radial frond with more than one p-vertex, consider the p-subtree $G_{v}$ of a p-vertex $v$ in such a frond; the nonradial fronds of $G$ are those of $G_{v}$. Otherwise, let $S$ be the multiset of nonradial fronds occurring in the p-subtrees. Let $T$ be the multiset of nonradial fronds occurring in the radial fronds with the p-vertex deleted. Subtract $T$ from $S$.

Case 1b: all subtrees in $D$ have diameter $d$ and $G$ is bicentral. Proceeding as in case 1a the fronds with their bicenter marked may be determined. Let $v$ be a p-vertex in a radial frond with $j$ p-vertices, on the side with the smaller number of such fronds, or either side if there are the same number on both sides. There is an extra frond in $G_{v}$, and a missing one; the side on which the extra frond should be replaced is determined.

Case 2a: there is one subtree in $D$ of diameter $d-1$ and $G$ is central. This occurs iff $G$ has two radial fronds, one with one p-vertex (call it $L$ ), and another with $j>1$ p-vertices (call it $M$ ). The p-subtrees are the one of diameter $d-1$, together with those of diameter $d$ whose number of p-vertices is least. The nonradial fronds are determined from any p-subtree of diameter $d$. Let $w$ be the closest vertex in $M$ to the center which has degree greater than 2. Let $e$ range over the edges incident to $w$, other than the edge on the path to the center. Call the subtree consisting of all paths from $w$ to a leaf of $M$ containing a given 
$e$ a subfrond. Let $M_{s}$ for $1 \leq s \leq j$ be the subfronds. Suppose $j>2$. In each psubtree, $L$ and all but one of the $M_{s}$ are determined. The entire multiset of the $M_{s}$ is determined by taking the union and dividing by $j$. Suppose $j=2$. The multisets $\left\{M_{1}, L\right\}$ and $\left\{M_{2}, L\right\}$ are determined by the p-subtrees of diameter $d$, and $\left\{M_{1}, M_{2}\right\}$ by the p-subtree of diameter $d-1$. It readily follows that $M_{1}$, $M_{2}$, and $L$ are determined.

Case 2b: there is one p-subtree of diameter $d-1$ and $G$ is bicentral. This occurs iff $G$ has one radial frond on one side, and $j>1$ p-vertices on the second side. There may be more than one frond on the second side; let $k$ be the number. The p-subtrees are as in case 2a. The nonradial fronds are determined as in case 2a. Suppose $j>2$. If $k>1$ the radial fronds and $L$ are determined from the p-subtrees of diameter $d$, by usual arguments. If $k=1$ the subfronds and $L$ are determined from the p-subtrees of diameter $d$, as in case 2a. Suppose $j=2$. If $k=2$ then $L$ and the fronds on the second side are determined from the diameter $d$ p-subtrees. If $k=1$ the argument is as in case 2a.

Case 3: there are two subtrees in $D$ of diameter $d-1$, and the rest have diameter $d$. This is the case iff $G$ is central and there are two fronds. Let $S$ be one of the p-subtrees. It contains a unique path if length $d-1$, and both endpoints are degree $1 . G$ is obtained by adding an edge at one of the endpoints. It is easily seen that which end is either determined or irrelevant. 